\title{
Clinical characteristics of children with congenital anomalies of the kidney and urinary tract and predictive factors of chronic kidney disease
}

\author{
Pınar Gür Çetinkaya ${ }^{1 \oplus}$, Bora Gülhan ${ }^{2 \oplus}$, Ali Düzova ${ }^{2 \oplus}$, Nesrin Beşbaş ${ }^{2}$, \\ Mutlu Hayran ${ }^{3 \oplus}$, Rezan Topaloğlu' ${ }^{2 \oplus}$, Fatih Özaltın ${ }^{2,4,5 \odot}$ \\ ${ }^{2}$ Division of Pediatric Nephrology, ${ }^{1}$ Department of Pediatrics and ${ }^{3}$ Department of Preventive Oncology, ${ }^{4}$ Nephrogenetics Laboratory, \\ Hacettepe University Faculty of Medicine, Ankara; ${ }^{5}$ Hacettepe University Center for Biobanking and Genomics, Ankara, Turkey.
}

\begin{abstract}
Background. Congenital anomalies of kidney and urinary tract (CAKUT) are the leading causes of chronic kidney disease (CKD) in childhood. Determining the clinical course, outcome, and prognostic factors of this heterogeneous disease group is important to provide appropriate management and follow-up. Therefore, we aimed to identify the risk factors of CKD in CAKUT and the differences in clinical courses between disease subgroups.
\end{abstract}

Methods. Three hundred patients (M/F: 203/97) divided into 16 CAKUT categories were enrolled in the study. Logistic regression and survival analyses were performed to determine the risk factors for CKD that is defined as estimated GFR (eGFR) lower than $90 \mathrm{ml} / \mathrm{min} / 1.73 \mathrm{~m}^{2}$ for at least 6 months.

Results. The median age of the study population at the time of the diagnosis was 0.6 years (IQR; 0.1-4.0 years). Among available prenatal diagnoses $(n=138)$, hydronephrosis $(\mathrm{HN})(\mathrm{n}=83 ; 60.1 \%)$ and multicystic dysplastic kidney (MCDK) $(n=39 ; 28.2 \%)$ were the most frequently encountered ones. A total of 24 patients had CKD, and 13 of them (54.1\%) progressed to end stage renal disease (ESRD). Patients with posterior urethral valve (PUV) had CKD and ESRD more frequently when compared to the other diagnostic groups ( $\mathrm{p}<0.001$ for CKD, and $\mathrm{p}<0.001$ for ESRD). Furthermore, the PUV subgroup progressed to ESRD (median 3.63 years) earlier than the other subgroups. The diagnosis of PUV, proteinuria on the first admission, vesicoureteral reflux, and oligohydramnios were identified as independent predictors for CKD in the multivariate logistic regression analysis.

Conclusions. Knowing predictive factors for CKD in patients with CAKUT is valuable for physicians in order to determine appropriate treatment strategies and prognosis.

Key words: CAKUT, children, chronic kidney disease, risk factors.

Congenital anomalies of kidney and urinary tract (CAKUT) are one of the most common causes of chronic kidney disease (CKD), accounting for $30 \%$ of the pediatric CKD population. CAKUT occurs in approximately 3-6 per 1,000 live births, ${ }^{1}$ presents an increased risk for CKD, and thus constitutes the most frequent cause of

Fatih Özaltın

fozaltin@hacettepe.edu.tr

Received 5th October 2018, revised 17th November 2019, 8th December 2019, 3rd February 2020,

accepted 16th February 2020. the end stage renal disease (ESRD) requiring renal replacement therapy for survival in children. ${ }^{2}$ CAKUT can be diagnosed as early as 18-20 gestational weeks, with the most common prenatal finding being oligohydramnios. ${ }^{1}$ It has a broad phenotypic spectrum that consists of many subgroups with different clinical severity and prognosis. It is important to know the clinical presentations and differences in the clinical courses of CAKUT subgroups and to identify the predisposing factors to CKD in order to initiate appropriate treatment in a timely manner. We aimed to determine the risk 
factors for CKD in patients with CAKUT, the differences in respect to the clinical courses, the frequencies of subgroups, and any additional unfavorable impact of CAKUT on the patients.

\section{Material and Methods}

This retrospective study consisted 300 patients with the diagnosis of "Congenital anomalies of the kidney and urinary tract (CAKUT)" between years 2000-2016. All patients with CAKUT, except for isolated vesicoureteral reflux (VUR), were enrolled in the study. The diagnosis of CAKUT was based on renal ultrasonography, 99m-dimercaptosuccinic acid (DMSA), 99m-diethylenetriamine pentaacetic acid (DTPA) or Tc-99m-mercaptoacetyltriglycine (MAG3) radionuclide scanning and/or voiding cystourethrography (VCUG). Of the patients, 149 had DMSA, 101 had MAG3, 46 had DTPA scanning, and 67 had VCUG either in our hospital or elsewhere. VUR was grouped into low (i.e. grades 1, 2, and 3) or high (i.e. grades 4 and 5). The study population was grouped into 16 categories (Table I). The diagnosis groups of posterior urethral valve (PUV), multicystic dysplastic kidney (MCDK), renal agenesis (RA), hydronephrosis $(\mathrm{HN})$, ureteropelvic junction obstruction (UPJO), and the others which are composed of other diagnosis subgroups of CAKUT, were included in the survival analysis and risk factor analysis for CKD. Patients with hydronephrosis determined by antenatal ultrasonography were defined as antenatal hydronephrosis (antenatal HN). ${ }^{3}$ Antenatal $\mathrm{HN}$ and $\mathrm{HN}$ corresponded to non-obstructive and non-refluxing renal pelvis dilatation. All available data including renal ultrasonography, DMSA, DTPA/MAG3 scanning, VCUG, serum biochemistry, history of urinary tract infections (UTI), antibiotic prophylaxis, urological interventions, proteinuria on the first admission, and the follow-up periods were obtained from the hospital records. Estimated glomerular filtration rate (eGFR) was calculated with the Schwartz formula $(k=0.33$ for premature infants $<1$ year old; $\mathrm{k}=0.45$ for full term infants $<1$ year old; $\mathrm{k}=0.55$ for all boys and girls aged 2-12 years and girls aged 13-21 years, ; $k=0.70$ for boys aged 13-21 years). ${ }^{4}$ CKD was defined as eGFR below $90 \mathrm{ml} / \mathrm{min} / 1.73 \mathrm{~m}^{2}$ for at least 6 months. Staging of CKD was based on "KDIGO criteria" 5 according to eGFR values as stage $2(89-60 \mathrm{ml} /$ $\left.\mathrm{min} / 1.73 \mathrm{~m}^{2}\right)$, stage $3\left(59-30 \mathrm{ml} / \mathrm{min} / 1.73 \mathrm{~m}^{2}\right)$, stage $4\left(29-15 \mathrm{ml} / \mathrm{min} / 1.73 \mathrm{~m}^{2}\right)$, and stage 5 (ESRD, $<15 \mathrm{ml} / \mathrm{min} / 1.73 \mathrm{~m}^{2}$ ). Specific diagnostic criteria $^{6,7}$ were used for the children younger than 2 years and neonates for CKD diagnosis during enrollment. All the patients were evaluated for prematurity, CKD, proteinuria, and UTI. Midstream urine samples after appropriate perineal cleaning were tested, and urine culture was performed to diagnose UTI in the patients with urinary tract symptoms for older children. Urine was collected by urethral catheterization or suprapubic aspiration in infants and neonates. Proteinuria was defined as $>0.2 \mathrm{mg} / \mathrm{mg}$.creatinine of a urinary protein/ creatinine ratio $(\mathrm{Up} / \mathrm{Uc})$ for children older than 2 years $^{8}$ and $>0.5 \mathrm{mg} / \mathrm{mg}$ for children younger than 2 years. ${ }^{9}$ Children who did not attend the follow-up visits regularly, who did not have long term follow-up periods or who we were not able to contact by telephone were excluded from the survival analyses.

All procedures in this retrospective study were performed in accordance with the ethical standards of the institutional ethics committee (GO 13/166-27) and with the 1964 Helsinki declaration and its later amendments or comparable ethical standards. Informed consent forms were obtained from all individual participants included in the study.

\section{Statistical Analysis}

Statistical Package for the Social Science (SPSS) version 18.0 was used for data analysis. A Chi-square test was applied for assessing the demographic features of the patients. Mean values, medians, interquartile ranges (IQR), and standard deviations (SD) were calculated based on the clinical and laboratory results. Chisquare, Fishers exact, and Yates correction tests were used to analyze the differences between groups. Mann-Whitney U and Kruskal Wallis 
tests for continuing variables and the Wilcoxon Signed Rank test for dependent variables were applied. Possible risk factors for CKD identified with univariate analyses were included in the logistic regression analysis to determine the independent predictors of the patient result. Hosmer-Lemeshow goodness of fit statistics was used to assess model fit. Survival analysis of diagnosis groups during the follow-up period regarding the progression to CKD was assessed by Kaplan Meier analysis. A p-value less than 0.05 was considered statistically significant.

\section{Results}

A total of 300 children (M/F: 203/97) with the diagnosis of CAKUT were included (Table I). The median age at the first admission was 0.6 years (IQR; 0.1-4.0 years), the mean duration of the follow-up was $5.7 \pm 3.9$ years, and the mean age at the last visit was $8.7 \pm 6.1$ years. Of the patients, $138(46 \%)$ were diagnosed in the antenatal period at a mean gestational week of $25.5 \pm 5.8$. Among this group, $\mathrm{HN}(\mathrm{n}=83 ; 60.1 \%)$ and MCDK $(n=39 ; 28.2 \%)$ were the two leading diagnoses. While the diagnosis of antenatal HN was confirmed in 67 out of 83 patients with postnatal USG, initial prenatal diagnosis was changed in 16 patients after the delivery as follows: UPJO in seven, normal in six, MCDK in two patients, and renal agenesis in one patient (Fig. 1).

Of 67 patients who had VCUG, 24 had no reflux, 43 had reflux (30 low grade, 13 high grade). Twelve out of 43 patients with VUR (28\%) also had diagnosis of PUV. Of 20 patients with PUV, 7 had high grade VUR (35\%), 5 had low grade VUR (25\%). UTI was frequent in the high grade VUR group compared to the low grade VUR group $(p=0.031)$. Rate of proteinuria at the first admission was similar in patients with VUR $(7 / 43,16.3 \%)$ and without $(6 / 24,25 \%)$ $(p=0.527)$. Similarly, rate of proteinuria at the first admission was also comparable in patients with high and low grade VUR (15.4\% vs $16.7 \%$, respectively, $\mathrm{p}=0.648)$. The frequency of $\mathrm{CKD}$ was $33.3 \%$ (10/30) in low grade VUR and $38.5 \%$ $(5 / 13)$ in high grade VUR patients $(p=0.742)$. Diagnoses were as follows in patients with low grade VUR and CKD: PUV ( $n=5), \operatorname{MCDK}(n=1)$, RA ( $n=1), H N(n=1)$, UPJO ( $n=1)$, horse shoe kidney $(n=1)$ and in patients with high grade VUR and CKD: PUV $(n=4)$, RA $(n=1)$. A total of 149 patients $(49.7 \%)$ who underwent $99 \mathrm{mTc}$ DMSA; 49 (32.8\%) had unilateral, 4 (2.6\%) had bilateral renal scarring.

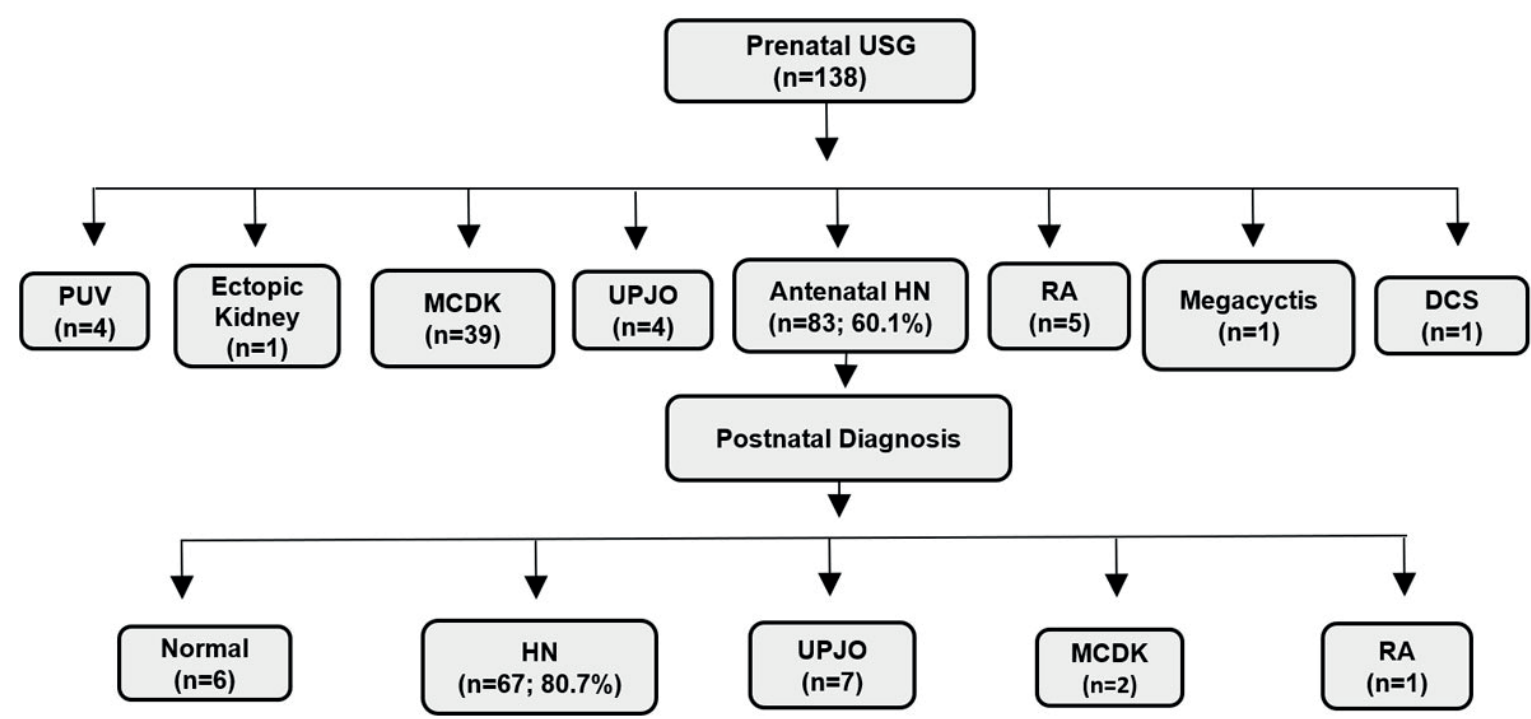

Fig. 1. Change of Prenatal Diagnosis of the Study Group in Postnatal Period

USG: ultrasonography, PUV: posterior urethral valve, MCDK: multicystic dysplastic kidney, HN: hydronephrosis, UPJO: obstruction of ureteropelvic junction, RA: renal agenesis, DCS: double collecting system 


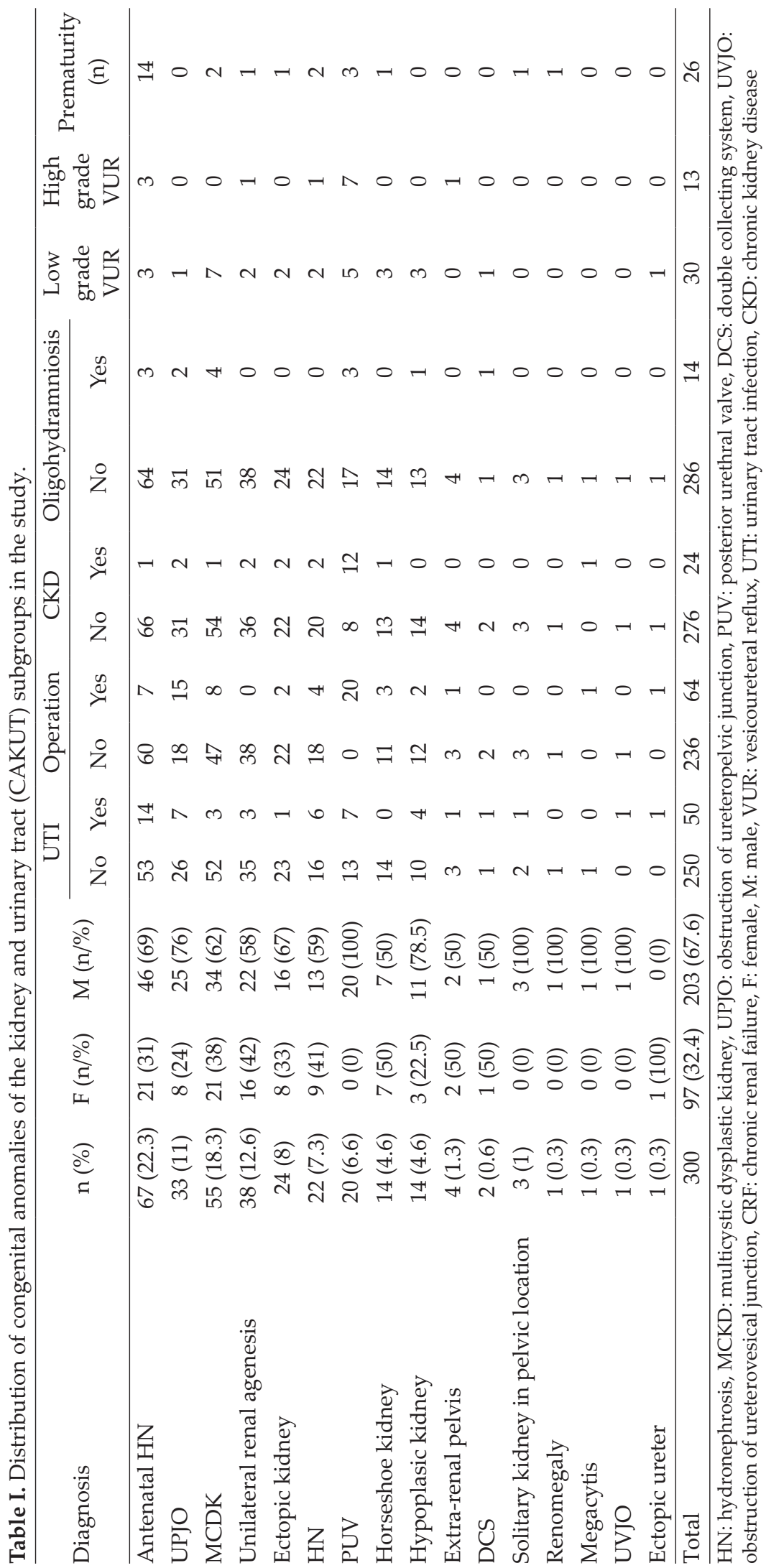


We evaluated the relationship between VUR, UTI and CKD. Of 43 patients with VUR, 15 had CKD (35\%), and 10 of these CKD patients had ESRD (66.6\%). Patients with VUR experienced CKD and ESRD more significantly than the patients without VUR ( $p<0.001$ for CKD, $p$ $<0.001$ for ESRD). VUR was found to be an independent risk factor for CKD in multivariate logistic regression analysis [OR 3.642; 95\% CI 1.073-12.355, $\mathrm{p}=0.038$ ]. Although CKD occurred more in patients with UTI ( $p=0.043$ ), albeit the ratio of ESRD was not different between the patients with or without UTI ( $p=0.373)$, UTI was not found an independent risk factor for CKD. Patients with bilateral renal scarring progressed to CKD more frequently than patients with unilateral renal scarring $(p=0.001)$. However, having bilateral renal parenchymal scarring was not found an independent risk factor for CKD in multivariate regression analysis.

Sixty-four patients $(21.3 \%)$ had at least one urological operation, 39 of which occurred in the first year of life. Valve ablation was performed in all patients with PUV $(n=20)$. Of the CKD group, 18 patients $(75 \%)$ underwent urological operations. Diagnoses were as follows: PUV $(n=12)$, UPJO $(n=2)$, horseshoe kidney with neurogenic bladder $(n=1)$, megacyctis $(n=1)$, $\mathrm{HN}(\mathrm{n}=1)$, and ectopic kidney with neurogenic bladder $(n=1)$. A nephrectomy was performed to the non-functional kidney in 15 patients [MCDK $(n=6), H N(n=2)$, ectopic kidney $(n=$ $2)$, PUV $(n=1)$, ectopic ureter $(n=1)$, UPJO $(n=$ $1)$, antenatal $\mathrm{HN}(\mathrm{n}=1)$, and extrarenal pelvis $(n=1)$ ] due to recurrent UTI despite antibiotic prophylaxis, recurrent urolithiasis, or treatmentresistant hypertension. Patients with PUV and UPJO more significantly underwent urological intervention in the first year of life (PUV vs other subgroups, $\mathrm{p}=0.002$, UPJO vs other subgroups; $p=0.001$ ). Patients with oligohydramnios had more urological interventions compared to the subjects without $(\mathrm{p}<0.001)$. Patients who underwent surgical operations had an increased incidence of UTI (71.9\%) and received more frequent antibiotic prophylaxis $(57.9 \%)$ compared to the non-operated patients (UTI; $\mathrm{p}=$ 0.006 and antibiotic prophylaxis; $\mathrm{p}<0.001)$.
The diagnosis of PUV, oligohydramnios, VUR, and proteinuria on the first admission were the independent risk factors for CKD in multivariate logistic regression analysis (Table II). Among the other disease subgroups, the diagnosis of PUV independently conferred a worse prognosis in survival analysis as well (Fig. 2). There were 24 patients with the diagnosis of CKD [stage $2(\mathrm{n}=6)$, stage $3(\mathrm{n}=3)$, stage $4(\mathrm{n}=$ $2)$, stage $5(n=13)(E S R D)]$. Both CKD and ESRD were observed more frequently in the PUV subgroup than the other diagnosis groups ( $p$ $<0.001)$. PUV was an independent predictor for CKD [OR 6.518; 95\% CI 1.573-27.003, p= 0.01)]. Furthermore, patients with PUV progressed to ESRD (median 3.63 years) earlier than the other subgroups. Eleven patients [PUV $(n=7)$, unilateral renal agenesis $(n=2), \operatorname{MCDK}(n=1)$, and horseshoe kidney with neurogenic bladder $(n=1)$ ] received dialysis treatment, and 7 of them [PUV $(n=5)$, renal agenesis $(n=1)$, and horseshoe kidney with neurogenic bladder $(n=1)$ ] underwent renal transplantation. The median eGFR values at the last visit and follow-

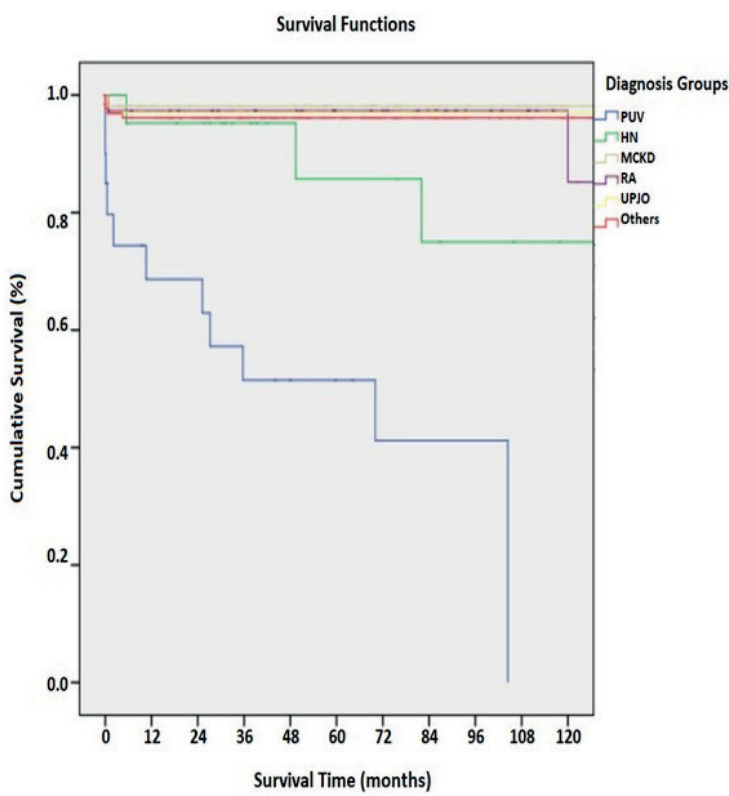

Fig. 2. The Follow Up Period up to the Time of CKD in Months Based on Congenital Anomalies of the Kidney and Urinary Tract (CAKUT) Phenotypes

PUV: posterior urethral valve, MCKD: multicystic dysplastic kidney, HN: hydronephrosis, UPJO: obstruction of ureteropelvic junction 
Table II. Predictive factors of chronic kidney disease in CAKUT.

\begin{tabular}{lcccccc}
\hline \multirow{2}{*}{ Predictive Factors for CKD } & \multicolumn{3}{c}{ Univariate } & \multicolumn{3}{c}{ Multivariate } \\
\cline { 2 - 7 } & OR & $95 \%$ CI & $\mathrm{p}$ & OR & $95 \%$ CI & $\mathrm{p}$ \\
\hline VUR & 13.192 & $5.128-33.940$ & 0.001 & 3.642 & $1.073-12.355$ & 0.038 \\
Oligohidramnios & 17.933 & $5.568-57.757$ & 0.001 & 11.672 & $2.380-57.242$ & 0.002 \\
UTI & 2.222 & $0.869-5.678$ & 0.095 & & & \\
Prematurity & 4.518 & $1.603-12.730$ & 0.004 & & & \\
Diagnosis of PUV & 25.103 & $8.854-71.169$ & $<0.001$ & 6.518 & $1.573-27.003$ & 0.010 \\
Gender & 3.674 & $1.069-12.632$ & 0.039 & & & \\
Bilateral paranchymal scar & 5.186 & $1.980-13.578$ & $<0.001$ & & & \\
Proteinuria at first admission & 4.518 & $1.603-12.730$ & 0.004 & 11.193 & $2.310-54.235$ & 0.003 \\
\hline
\end{tabular}

CKD: chronic kidney disease, OR: odds ratio, CI: confidential interval, VUR: vesicoureteral reflux, UTI: urinary tract infection, PUV: posterior urethral valve, HN: hydronephrosis.

up period after renal transplantation of these seven patients were $89.5 \mathrm{ml} / \mathrm{min} / 1.73 \mathrm{~m}^{2}$ (IQR: $22.1-101.0 \mathrm{ml} / \mathrm{min} / 1.73 \mathrm{~m}^{2}$ ) and 116.0 months (101.0-136.0 months), respectively. One patient with PUV and the patient with renal agenesis progressed to CKD after renal transplantation, whereas other five patients had normal graft functions at the time of writing of this report. Those patients who progressed to CKD after kidney transplantation received deceased donor kidneys. Graft losses were due to non-compliance to the immunosuppressive medications in the PUV patient and BK nephropathy in the other. Patients with oligohydramnios $(n=14)$ had bilateral renal and urinary tract anomalies. These patients progressed to CKD more frequently $(\mathrm{n}=7 / 14$, $50 \%$ vs $\mathrm{n}=17 / 286$ ) and earlier [9.62 (IQR 2.3259.63) months vs 59.72 (IQR 6.80-120.01) months] than the patients without oligohydramnios ( $p<0.001$ for both). Similarly, ESRD observed earlier in patients with oligohydramnios than those without. The median age of the patients with ESRD and oligohydramnios $(n=5)$ was 3.31 months (IQR; 0.00-55.37 months) whereas the median age of the patients with ESRD without oligohydramnios $(\mathrm{n}=8)$ was 56.82 months (IQR; 0.10-83.79 months). In multivariate analysis, oligohydramnios was found as an independent risk factor for CKD [OR 11.672; 95\% CI 2.38057.242, $\mathrm{p}=0.002)]$. There were 26 patients who were born prematurely $(8.6 \%)$ with a median gestational age of 35 weeks (IQR: 33-36 weeks).
There was no difference between the patients with or without prematurity in terms of eGFR values on the first admission and at the last visit ( $p=0.577$ on the first admission, $p=0.252$ at the last visit). Prematurity was significantly observed in patients with oligohydramnios $(\mathrm{p}=$ $0.025)$ and CKD $(p=0.002)$, but not in patients with ESRD $(p=0.318)$. Prematurity was not found an independent predictive factor for CKD. Fifteen patients had proteinuria on the first admission. The diagnosis of these patients were as follows; PUV $(n=6)$, UPJO $(n=3), \operatorname{RA}(n=$ $2), \operatorname{MCDK}(n=1), \operatorname{DCS}(n=1), \operatorname{AHN}(n=1)$, and $\mathrm{HN}(\mathrm{n}=1)$. Of these patients, eight had CKD and five of them progressed to ESRD. Additionally, having proteinuria on the first admission was an independent risk factor for CKD regardless of CAKUT subtype [OR 11.193; 95\% CI 2.310$54.235, \mathrm{p}=0.003)]$.

\section{Discussion}

CAKUT consists of a heterogeneous group of disorders, and the clinical courses of these subgroups are extremely different. ${ }^{10}$ Most of the patients with CAKUT often progress to ESRD at a slower rate; however, certain subgroups do progress more rapidly. ${ }^{10}$ In this study, we focused on the differences regarding the prognosis of each subgroup and the factors influencing the progression to CKD. The current study indicates that the prognosis is highly dependent on the CAKUT phenotype. 
In different CAKUT subgroups, PUV was found to be an independent risk factor for CKD as underlined in previous reports. ${ }^{11}$ Besides PUV, VUR, proteinuria on the first admission, and oligohydramnios were also found to be associated with CKD in our study.

PUV leads to bilateral renal obstruction and renal dysplasia in addition to bladder dysfunction. ${ }^{12}$ It confers a poorer prognosis than the other frequently seen obstructive uropathy, UPJO, which tends to occur unilaterally and have a milder phenotype. ${ }^{13} \mathrm{PUV}$ is the leading cause of CKD in newborn males, ${ }^{14}$ and ESRD occurs in a wide range between 5\% and 64\% in the reports. ${ }^{15}$ We found that CKD and ESRD were observed in $55 \%$ and $40 \%$ of the patients with PUV, respectively. A possible reason for these high ratios is likely to be due to the longer follow-up period of our patients, and the nature of our hospital (i.e. a tertiary referral clinical center). In our study, $40 \%$ of our patients with PUV progressed to ESRD despite relatively early surgical ablation This indicates that CKD in this group of patients is not solely due to mechanical obstruction itself but accompanying renal dysplasia, which is expected in this patient population. ${ }^{12}$

In the current study, the patients with oligohydramnios progressed to CKD in a shorter follow-up period than those who do not have. Oligohydramnios indicates severe abnormality in the urinary system in the antenatal period..$^{16,17}$ Therefore, oligohydramnios is mostly associated with renal impairment, poor prognosis, and ESRD. ${ }^{16,17}$ Klaassen et al. ${ }^{17}$ revealed that all infants with oligohydramnios developed CKD, and $40 \%$ of these individuals progressed to ESRD in the first 3 months of life. In our study group, patients with oligohydramnios progressed to CKD and ESRD at a ratio of $50 \%$ and $36 \%$, respectively. Additionally, those patients progressed to CKD at a mean age of 9.62 months. As we could not reach the prenatal information of all patients, the proportion of CKD and ESRD in patients with oligohydramnios would most likely be higher. In agreement with previous reports, ${ }^{16,17}$ our patients with oligohydramnios experienced ESRD and underwent surgical interventions more frequently than the patients without. In addition, having oligohydramnios was identified as an independent risk factor for CKD in our study. Taken together, oligohydramnios should be considered as one of the earliest markers of CKD in patients with CAKUT.

Inourstudy, although we did not find statistically significant difference between low and high grade VUR in terms of CKD, presence of VUR has been identified as an important risk factor in the course of CKD as shown previously. ${ }^{18}$ The impact of VUR on renal survival is controversial. In some of the studies, ${ }^{11}$ VUR has been associated with poor renal survival, but on the contrary, the others have established better results. ${ }^{19}$ In the literature, it has been shown that VUR accompanies with PUV at a ratio of $50 \%{ }^{20}$ Similar to existing literature data, in our study, $60 \%$ of PUV patients had VUR, and $35 \%$ of them were high grade. The relation between VUR and CKD identified in the current study may be explained by the frequent association of VUR with PUV, which was established as main cause of CKD in CAKUT. This may also explain no difference between low and high grade VUR in terms of CKD as most of the CKD patients had PUV diagnosis, which was considered the main cause for CKD. In the previous reports, UTI has been frequently observed in patients with CAKUT. ${ }^{21}$ Recurrent UTI rarely leads to CKD without any underlying renal abnormalities ${ }^{22}$ and has been shown to worsen the prognosis of the patients with congenital kidney diseases and bilateral renal impairment. ${ }^{23}$ As noted in previous studies, CKD was significantly observed in patients who had recurrent UTI. However, we did not find UTI as a risk factor for the development of CKD in the current study. In contrast to published data, ${ }^{24}$ UTI did not influence the course of CKD or ESRD in our study population. Another important parameter of the progression to CKD is the presence of parenchymal scarring in patients with VUR. The existing UTI and inflammatory process are well-known reasons for renal scarring. ${ }^{25}$ The 
relevance of renal scarring in CKD has been shown, and the renal parenchymal scarring has been reported as a risk factor for CKD. ${ }^{18,26}$ Studies have investigated the effect of the scarring on renal survival; ${ }^{18,26}$ however, few studies have analyzed whether this scarring is unilateral or bilateral. ${ }^{27}$ In a detailed research, ${ }^{27}$ patients with bilateral renal scarring have been reported to have more pronounced creatinine elevation and lower GFR values. In the present study, we also showed that the bilateral renal scarring had a significant association with CKD in patients with CAKUT.

Proteinuria is considered an important predictor of CKD in $\mathrm{CAKUT}^{28}$ and it is prominent during the follow-up period in patients with chronic renal disease. ${ }^{29}$ In our study, proteinuria at the referral time was marked in patients with CKD. Proteinuria has also been shown as an unfavorable factor in renal survival in patients with CAKUT. ${ }^{11}$ In agreement with existing literature data, we demonstrated that proteinuria on the first admission was an independent predictor for CKD, and therefore should be monitored during follow-up.

Prematurity was another parameter that also significantly associated with patients who had CKD. In preterm infants, the size and the number of the glomeruli and GFR levels are significantly diminished compared to full-term babies. ${ }^{30}$ Having a baseline reduced number of nephrons in premature infants may contribute to the ongoing programmed cell-death in CAKUT, and this may facilitate the progression to CKD. There are conflicting data in the literature regarding prematurity and CKD. It has been reported that, preterm infants have normal renal functions. ${ }^{31}$ However, Melo et al. ${ }^{32}$ suggested that prematurity was an independent predictor for renal failure in CAKUT. In our study we did not find prematurity as a risk factor for CKD, but this would be related to the lack of full antenatal information of the study patients.
Our study has some limitations that usually occur in retrospective studies. We had missing data regarding prenatal ultrasonographic assessments. In addition, the number of patients in some subgroups was too small, and subgroups were extremely heterogeneous. In the current study, VCUG was not performed in all patients. Therefore, actual VUR ratio might be different than we found. We did not find bilateral renal scarring as an independent risk factor for CKD. This could be an artifact as we had very small number of patients having bilateral renal scarring $(n=4)$. Additional limitation of our study would be an unequal follow-up durations of the patients with different CAKUT subgroups. All of these factors restrict to make a clear comparison between the subgroups. Patient registries would overcome this inability as these registries yield more patients with rare CAKUT subgroups and sufficient follow-ups. On the other hand, our study was conducted in a broad pediatric CAKUT population that was monitored during a fairly long period.

In conclusion, the current study gives a general perspective about children with the diagnosis of CAKUT who applied to a tertiary clinical center in Turkey. Diagnosis of PUV, VUR, proteinuria on the first admission, and oligohydramnios have been identified as predictive factors for CKD. Renal prognosis of the patients highly depends on the CAKUT phenotype. PUV is associated with the poorest prognosis among the CAKUT subgroups. Surgical interventions seemed not to prevent but only postpone the process of CKD in the obstructive phenotypes of CAKUT. These factors may have clinical utility during management and estimate a long-term prognosis in patients with CAKUT.

\section{REFERENCES}

1. Yosypiv IV. Congenital anomalies of the kidney and urinary tract: a genetic disorder? Int J Nephrol 2012; 2012: 909083

2. Wühl E, van Stralen KJ, Verrina E, et al. Timing and outcome of renal replacement therapy in patients with congenital malformations of the kidney and urinary tract. Clin J Am Soc Nephrol 2013; 8: 67-74. 
3. Mallik M, Watson AR. Antenatally detected urinary tract abnormalities: more detection but less action. Pediatr Nephrol 2008; 23: 897-904.

4. Pierrat A, Gravier E, Saunders C, et al. Predicting GFR in children and adults: a comparison of the Cockcroft-Gault, Schwartz, and modification of diet in renal disease formulas. Kidney Int 2003; 64: 14251436.

5. Andrassy KM. Comments on'KDIGO 2012 clinical practice guideline for the evaluation and management of chronic kidney disease'. Kidney Int 2013; 84: 622-623.

6. Levin A, Stevens PE, Bilous RW, et al. Kidney Disease: Improving Global Outcomes (KDIGO) CKD Work Group. KDIGO 2012 clinical practice guideline for the evaluation and management of chronic kidney disease. Kidney Int Suppl 2013; 3: 1-150.

7. Zaritsky JJ, Warady BA. Chronic kidney disease in the neonate. Clin Perinatol 2014; 41: 503-515.

8. Ariceta G. Clinical practice: proteinuria. Eur J Pediatr 2011; 170: 15-20.

9. Dale-Shall AW, Feld LG. Approach to the child with proteinuria. In: Elzouki AY, Harfi HA, Nazer HM, Oh W, Stapleton FB, Whitley RJ (eds). Textbook of Clinical Pediatrics (2nd ed). (6 Volume Set) Berlin: Springer-Verlag, 2012: 2711-2721.

10. Harambat J, van Stralen KJ, Kim JJ, Tizard EJ. Epidemiology of chronic kidney disease in children. Pediatr Nephrol 2012; 27: 363-373.

11. Sanna-Cherchi S, Ravani P, Corbani V, et al. Renal outcome in patients with congenital anomalies of the kidney and urinary tract. Kidney Int 2009; 76: 528-533.

12. Edouga D, Hugueny B, Gasser B, Bussières L, Laborde K. Recovery after relief of fetal urinary obstruction: morphological, functional and molecular aspects. Am J Physiol Renal Physiol 2001; 281: F26-F37.

13. Donovan BO, Ramji F, Frimberger D, Kropp BP. Neonatal urologic emergencies. In: Gearhart JP, Pink R, Mouriquand P, (eds). Pediatric Urology (2nd edt). 2010: 709-719.

14. Warshaw BL, Edelbrock HH, Ettenger RB, et al. Renal transplantation in children with obstructive uropathy. J Urol 1980; 123: 737-741.

15. Roth KS, Carter WH, Chan JC. Obstructive nephropathy in children: long-term progression after relief of posterior urethral valve. Pediatrics 2001; 107: 1004-1010.

16. Mehler K, Beck BB, Kaul I, Rahimi G, Hoppe B, Kribs A. Respiratory and general outcome in neonates with renal oligohydramnios-a single-centre experience. Nephrol Dial Transplant 2011; 26: 3514-3522.
17. Klaassen I, Neuhaus TJ, Mueller-Wiefel DE, Kemper MJ. Antenatal oligohydramnios of renal origin: longterm outcome. Nephrol Dial Transplant 2007; 22: 432-439.

18. Chen MJ, Cheng HL, Chiou YY. Risk factors for renal scarring and deterioration of renal function in primary vesico-ureteral reflux children: a long-term follow-up retrospective cohort study. PloS One 2013; 8: e57954.

19. Ishikura K, Uemura O, Hamasaki $Y$, et al; Pediatric CKD Study Group in Japan in conjunction with the Committee of Measures for Pediatric CKD of the Japanese Society for Pediatric Nephrology Insignificant impact of VUR on the progression of CKD in children with CAKUT. Pediatr Nephrol 2016; 31: 105-112.

20. Nasir AA, Ameh EA, Abdur-Rahman LO, Adeniran JO, Abraham MK. Posterior urethral valve. World J Pediatr 2011; 7: 205-216.

21. Rodriguez MM. Congenital anomalies of the kidney and the urinary tract (CAKUT). Fetal Pediatr Pathol 2014; 33: 293-320.

22. Salo J, Ikäheimo R, Tapiainen T, Uhari M. Childhood urinary tract infections as a cause of chronic kidney disease. Pediatrics 2011; 128: 840-847.

23. Tasic V, Ristoska-Bojkovska N, Gucev Z, Lozanovski VJ. Poststreptococcal glomerulonephritis in children with congenital anomalies of the kidney and urinary tract. Ren Fail 2015; 37: 1440-1443.

24. Lee KH, Gee HY, Shin JI. Genetics of vesicoureteral reflux and congenital anomalies of the kidney and urinary tract. Investig Clin Urol 2017; 58(Suppl 1): S4-S13.

25. Olson PD, McLellan LK, Liu A, et al. Renal scar formation and kidney function following antibiotictreated murine pyelonephritis. Dis Mod Mech 2017; 10: 1371-1379.

26. Hiraoka M. Medical management of congenital anomalies of the kidney and urinary tract. Pediatr Int 2003; 45: 624-633.

27. Lahdes-Vasama T, Niskanen K, Rönnholm K. Outcome of kidneys in patients treated for vesicoureteral reflux (VUR) during childhood. Nephrol Dial Transplant 2006; 21: 2491-2497.

28. Neild GH. Life expectancy with chronic kidney disease: an educational review. Pediatr Nephrol 2017; 32: 243-248.

29. Lopez Pereira P, Espinosa L, Martinez Urrutina MJ, Lobato R, Navarro M, Jaureguizar E. Posterior urethral valves: prognostic factors. BJU Int 2003; 91 : 687-690. 
30. Rodríguez MM, Gómez AH, Abitbol CL, Chandar JJ, Duara S, Zilleruelo GE. Histomorphometric analysis of postnatal glomerulogenesis in extremely preterm infants. Pediatr Dev Pathol 2004; 7: 17-25.

31. Iacobelli S, Loprieno S, Bonsante F, Latorre G, Esposito L, Gouyon JB. Renal function in early childhood in very low birthweight infants. Am J Perinatol 2007; 24: 587-592.
32. Melo BF, Aguiar MB, Bouzada MCF, et al. Early risk factors for neonatal mortality in CAKUT: analysis of 524 affected newborns. Pediatr Nephrol 2012; 27: 965-972. 